\title{
The impact of the novel coronavirus pandemic on gastrointestinal operative volume in the United States
}

\author{
Amanda C. Purdy ${ }^{1}$ - Brian R. Smith ${ }^{2} \cdot$ Samuel F. Hohmann $^{3,4} \cdot$ Ninh T. Nguyen $^{2}$ (I) \\ Received: 26 January 2021 / Accepted: 28 March 2021 / Published online: 19 April 2021 \\ (c) The Author(s), under exclusive licence to Springer Science+Business Media, LLC, part of Springer Nature 2021
}

\begin{abstract}
Background In March 2020, the Surgeon General recommended limiting elective procedures to prepare for the COVID-19 surge. We hypothesize a consequence of COVID-19 is reduced operative volume across the country. We aim to examine changes in volume of common gastrointestinal operations during COVID-19, including elective, urgent/emergent, and cancer operations. We also evaluate if hospitals with more COVID-19 admissions were most impacted.

Methods The Vizient database was used to determine monthly operative volume from November 2019 to June 2020 for elective operations (hiatal hernia repairs, bariatric surgery), urgent operations (cholecystectomies, appendectomies, inguinal hernia repairs), and cancer operations (colectomies, gastrectomies, esophagectomies). COVID-19 admissions per hospital were also determined. November 2019-January 2020 was defined as "pre-COVID." The monthly reduction in volume from pre-COVID was calculated for each operation. The top quartile (25\%) of hospitals with the most COVID admissions were also evaluated separately from hospitals with fewer COVID cases. Data were analyzed using analysis of variance.

Results Data from 559 hospitals were analyzed. The volumes of all operations evaluated were significantly reduced during the pandemic except gastrectomies and esophagectomies for cancer. The greatest reduction in all operations was in April. In April, the volume of bariatric surgery reduced by $98 \%(P<0.001)$, hiatal hernia repairs by $96 \%(\mathrm{P}<0.001)$, urgent cholecystectomies by $42 \%(P<0.001)$, urgent inguinal hernia repairs by $40 \%(\mathrm{P}<0.001)$, urgent appendectomies by $24 \%(P<0.001)$, and colectomies for cancer by $39 \%(P<0.001)$. Hospitals with the most COVID-19 admissions had greater reductions in all operations than hospitals with fewer COVID cases.

Conclusions The coronavirus pandemic led to a significant reduction in volume of all gastrointestinal operations evaluated except gastrectomies and esophagectomies. While elective, non-cancer operations were most affected, urgent and some cancer operations also declined significantly. As COVID-19 continues to surge, Americans may suffer continued limited access to surgical care and a significant operative backlog may be forthcoming.
\end{abstract}

Keywords COVID- $19 \cdot$ Surgery $\cdot$ Operative volume $\cdot$ Coronavirus $\cdot$ Pandemic

The novel coronavirus (COVID-19) pandemic has seemingly impacted every aspect of American lives since the

Ninh T. Nguyen

ninhn@hs.uci.edu

1 Department of Surgery, Harbor-University of California, Los Angeles Medical Center, Los Angeles, USA

2 Department of Surgery, Irvine Medical Center, University of California, 333 City Bldg. West, Suite 1600, Orange, CA 92868, USA

3 Center for Advanced Analytics and Informatics, Vizient, Irving, USA

4 Department of Health Systems Management, Rush University, Chicago, USA first reported case in the United States (US) on January 20th, 2020 [1]. The pandemic has spread across the country, directly affecting an astonishing number of Americans. As of January 19th, 2021, there have been over 23 million COVID infections and 394,395 COVID deaths reported in the United States [1]. Many Americans, even those that have not been infected with COVID-19 themselves, have seen their health indirectly affected by the pandemic because of challenges accessing care. We hypothesize that one of the consequences of the pandemic has been a decrease in gastrointestinal (GI) operative volume in the US.

On March 12th, 2020, the COVID-19 pandemic was declared a national emergency in the US [2]. At this time, the US was seeing reports of Italy, and becoming aware of 
just how deadly this virus could be. Cases were starting to increase in New York City, following Italy's trajectory. The public health strategy was to "flatten the curve" in an attempt to prevent everyone getting sick at the same time, which would overwhelm healthcare resources. There was an anticipated potential shortage of personal protective equipment, hospital beds, ventilators, and healthcare providers. In response to this, the American College of Surgeons issued a statement on March 13th urging hospitals and surgeons to limit elective procedures in order to avoid overwhelming our healthcare infrastructure [3]. Evidence was also emerging about the risks of patients with COVID-19 undergoing anesthesia, making a stronger case to delay non-urgent operations [4]. Surgeons faced difficult decisions on which scheduled cases could be delayed. On March 17th, the American College of Surgeons offered guidance for triaging scheduled procedures that took into account the medical urgency of the operation, the overall health of the patient, and the logistical feasibility of performing the operation [5].

Although there have been single-institution studies in the US that have shown a decrease in surgical volume during the pandemic, there is a lack of larger-scale studies [6]. Moving forward, it is important to quantify the impact of the COVID-19 pandemic on operative volume in the US. The aim of this study is to determine the changes in volume of common GI operations during the COVID-19 pandemic, including elective, urgent/emergent, and cancer operations. We also evaluate whether hospitals with the most COVID-19 admissions were disproportionally affected.

\section{Materials and methods}

Data were obtained using the Vizient clinical database [7]. The use of this database was approved by Vizient Inc. This study was considered exempt by the institutional review committee at the University of California, Irvine Medical Center as the Vizient database does not contain identifiable patient-level data. Patient informed consent was waived by the institutional review committee. The Vizient database contains data of all inpatients discharged from US academic medical centers that are members of Vizient and their affiliated hospitals. The data are based on billing records. The database reports only in-hospital outcome data and not postdischarge follow-up data. Data can be selected according to many criteria, including International Classification of Diseases (ICD) diagnosis code, ICD procedure code, and admission status. Admission statuses include emergency, urgent, and elective.

We queried the Vizient database using ICD-10 codes to determine the number of commonly performed elective operations (bariatric surgery and hiatal hernia repairs), cancer operations (colectomies, esophagectomies, and gastrectomies), and urgent/emergent operations (appendectomies, cholecystectomies, and inguinal hernia repairs) performed per month from November 2019 through June 2020. We also used the database to determine the total number of COVID-19 admissions at each hospital, using the ICD-10 code U071 for COVID-19. The $25 \%$ of hospitals with the most overall COVID-19 admissions were considered the "top COVID quartile."

The bariatric surgery cohort included patients with the diagnosis of obesity who underwent sleeve gastrectomy or gastric bypass. Hiatal hernia repairs included patients with the diagnosis of hiatal hernia and/or gastroesophageal reflux disease (GERD) who underwent hiatal hernia repair and/or fundoplication. Esophagectomies included patients with a diagnosis of esophageal cancer who underwent esophagectomy, and gastrectomies included patients with a diagnosis of gastric cancer who underwent complete or partial gastrectomy. Colectomies included patients with a diagnosis of colon cancer who underwent total or segmental colectomy. Appendectomies included urgent or emergent appendectomies done for appendicitis. Cholecystectomies included urgent or emergent cholecystectomies for cholecystitis. Inguinal hernia repairs included urgent or emergent inguinal hernia repairs for incarcerated and/or strangulated inguinal hernias.

We evaluated the monthly case volume trends for all hospitals and also separately for the hospitals in the top COVID quartile and the bottom COVID $75^{\text {th }}$ percentile. Case volumes were compared to the mean monthly case volumes from November 2019 through January 2020, which was defined as the "pre-COVID period." The percent reduction of cases was calculated for the month most affected to determine the greatest case volume reduction and also for June 2020 to determine if case volume was rebounding by June. Analysis of variance was used to determine if there was a significant difference in case volume based on month. $P$ value of less than 0.05 was considered significant.

\section{Results}

Data were obtained from 559 US hospitals. From November 2019 through June 2020, a total of 24,741 patients in the database underwent bariatric surgery, 8,919 underwent surgery for hiatal hernias, 17,824 underwent urgent or emergent cholecystectomy, 15,652 underwent urgent or emergent appendectomy, 2,743 underwent urgent or emergent inguinal hernia repair, 5,605 underwent partial or total colectomy for colon cancer, 1,166 underwent partial or total gastrectomy for gastric cancer, and 1,535 underwent esophagectomy for esophageal cancer. There was a total of 124,397 patients with COVID-19 in the database from November 2019 through June 2020. Hospitals had a median of 90 COVID-19 
cases (range 0-4,983; interquartile range 13-274). The hospitals in the "top COVID quartile" had 274 or more COVID admissions (Table 1).

The lowest volume of all GI operations evaluated was seen in April 2020. The elective operations saw the most significant decline. In April, bariatric operations were reduced by $98 \%(P<0.001)$ and hiatal hernia operations were reduced by $96 \%(P<0.001)$ (Fig. 1$)$. The volume of all urgent and emergent GI operations evaluated also significantly declined. In April, urgent cholecystectomies saw a $42 \%$ reduction $(P<0.001)$, urgent appendectomies saw a $24 \%$ reduction $(P=0.009)$, and urgent inguinal hernia repairs saw a $40 \%$ reduction $(P<0.001)$ (Fig. 2$)$. Of the cancer operations evaluated, all had the lowest volume in April, but only colectomies saw a statically significant decline. In April, colectomies for colon cancer were reduced by $39 \%$ $(P<0.001)$. Gastrectomies for gastric cancer were reduced by $14 \%(P=0.2)$, and esophagectomies were reduced by 17\% ( $P=0.7)$ (Fig. 3).

In April, hospitals in the top COVID quartile had a greater percent reduction of all cases than hospitals in the bottom $75^{\text {th }}$ percentile of COVID cases (Table 1). Although the reduction in gastrectomies per month at all hospitals was not statistically significant, there was a statistically significant decline in gastrectomies done for gastric cancer at hospitals in the top COVID quartile ( $32 \%$ reduction, $P<0.001)$.

Table 1 Total operations done per month by operation type

\begin{tabular}{|c|c|c|c|c|c|c|c|c|}
\hline & \multicolumn{6}{|c|}{ Total cases done per month, no. of cases } & \multirow{2}{*}{$\begin{array}{l}\% \text { Reduction from } \\
\text { Pre-COVID to Apr } \\
2020\end{array}$} & \multirow[t]{2}{*}{$P$-Value } \\
\hline & Pre-COVID ${ }^{\mathrm{a}}$ & Feb 2020 & Mar 2020 & Apr 2020 & May 2020 & Jun 2020 & & \\
\hline \multicolumn{9}{|l|}{ Bariatrics } \\
\hline Total & 4300 & 3671 & 2318 & 89 & 1736 & 4028 & $98 \%$ & $<0.001$ \\
\hline Top COVID Quartile & 2140 & 1939 & 1094 & 17 & 591 & 1769 & $99 \%$ & $<0.001$ \\
\hline Bottom COVID 75th Percentile & 2160 & 1732 & 1224 & 72 & 1145 & 2259 & $97 \%$ & $<0.001$ \\
\hline \multicolumn{9}{|l|}{ Hiatal Hernias } \\
\hline Total & 1544 & 1334 & 976 & 55 & 642 & 1279 & $96 \%$ & $<0.001$ \\
\hline Top COVID Quartile & 826 & 735 & 500 & 12 & 247 & 638 & $99 \%$ & $<0.001$ \\
\hline Bottom COVID 75th Percentile & 719 & 599 & 476 & 43 & 395 & 641 & $94 \%$ & $<0.001$ \\
\hline \multicolumn{9}{|l|}{ Cholecystectomies } \\
\hline Total & 2494 & 2412 & 2053 & 1451 & 2194 & 2232 & $42 \%$ & $<0.001$ \\
\hline Top COVID Quartile & 1136 & 1095 & 914 & 514 & 921 & 957 & $55 \%$ & $<0.001$ \\
\hline Bottom COVID 75th Percentile & 1358 & 1317 & 1139 & 937 & 1273 & 1275 & $31 \%$ & $<0.001$ \\
\hline \multicolumn{9}{|l|}{ Appendectomies } \\
\hline Total & 2095 & 1900 & 1880 & 1592 & 2028 & 1966 & $24 \%$ & 0.009 \\
\hline Top COVID Quartile & 934 & 872 & 817 & 645 & 887 & 943 & $31 \%$ & 0.04 \\
\hline Bottom COVID 75th Percentile & 1161 & 1028 & 1063 & 947 & 1141 & 1023 & $19 \%$ & 0.05 \\
\hline \multicolumn{9}{|l|}{ Inguinal Hernias } \\
\hline Total & 390 & 381 & 322 & 234 & 311 & 324 & $40 \%$ & $<0.001$ \\
\hline Top COVID Quartile & 181 & 182 & 140 & 93 & 140 & 171 & $49 \%$ & 0.003 \\
\hline Bottom COVID 75th Percentile & 209 & 199 & 182 & 141 & 171 & 153 & $33 \%$ & 0.003 \\
\hline \multicolumn{9}{|l|}{ Colectomies } \\
\hline Total & 783 & 799 & 777 & 481 & 534 & 664 & $39 \%$ & $<0.001$ \\
\hline Top COVID Quartile & 358 & 383 & 347 & 170 & 215 & 292 & $53 \%$ & $<0.001$ \\
\hline Bottom COVID 75th Percentile & 426 & 416 & 430 & 311 & 319 & 372 & $27 \%$ & $<0.001$ \\
\hline \multicolumn{9}{|l|}{ Gastrectomies } \\
\hline Total & 141 & 167 & 171 & 121 & 137 & 148 & $14 \%$ & 0.2 \\
\hline Top COVID Quartile & 66 & 99 & 97 & 45 & 63 & 83 & $32 \%$ & $<0.001$ \\
\hline Bottom COVID 75th Percentile & 74 & 68 & 74 & 76 & 74 & 65 & $-2 \%$ & 1.0 \\
\hline \multicolumn{9}{|l|}{ Esophagectomies } \\
\hline Total & 195 & 191 & 208 & 161 & 199 & 192 & $17 \%$ & 0.7 \\
\hline Top COVID Quartile & 98 & 86 & 95 & 64 & 98 & 95 & $35 \%$ & 0.4 \\
\hline Bottom COVID 75th Percentile & 97 & 105 & 113 & 97 & 101 & 97 & $0 \%$ & 1.0 \\
\hline
\end{tabular}

${ }^{a}$ Mean monthly cases done from November 2019 through January 2020 
6000

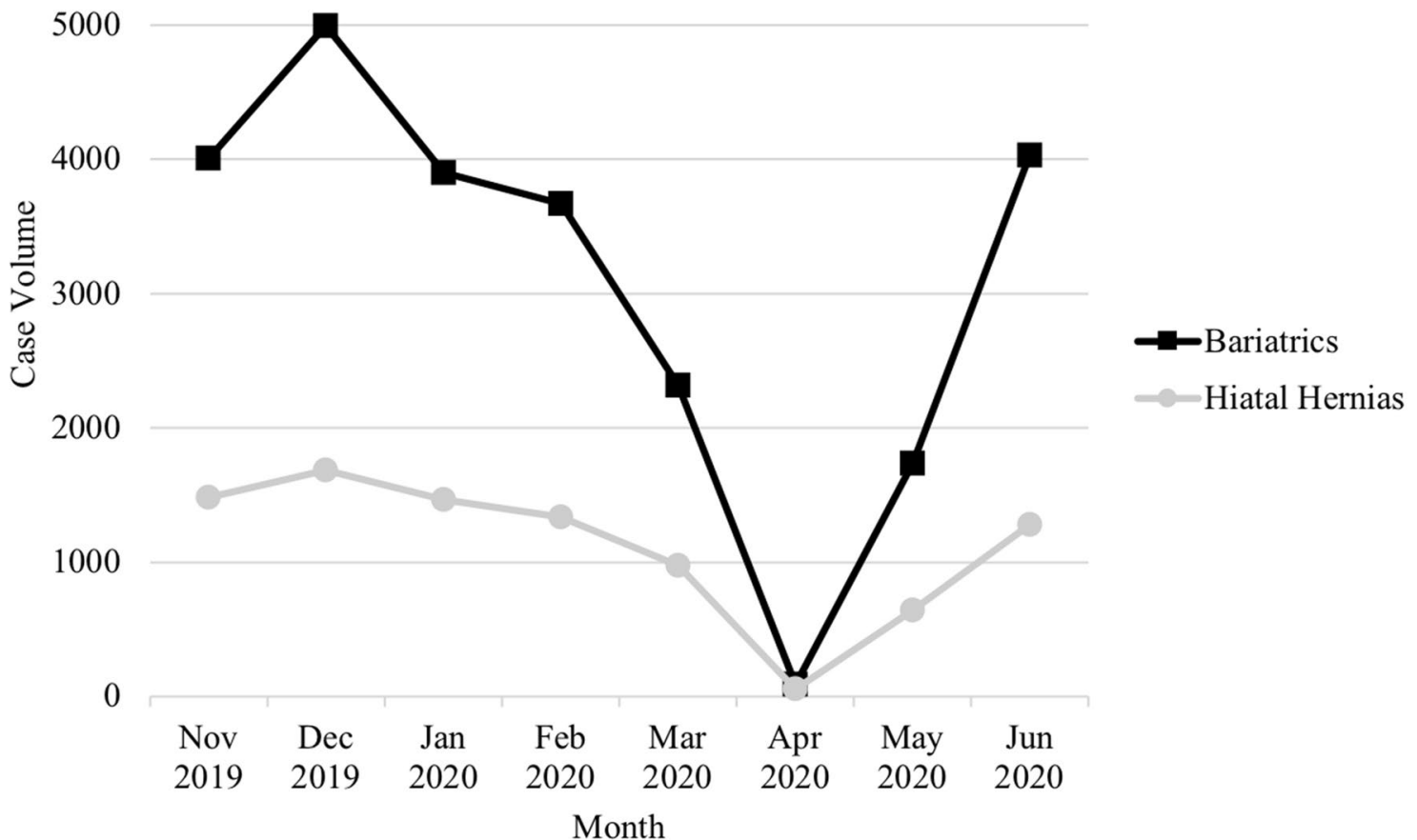

Fig. 1 Operative volume by month for elective operations

After April, operative volume for all GI operations evaluated began to increase (Figs. 1, 2, 3). In June 2020, compared to pre-COVID, there was a $6 \%$ reduction in bariatric operations, $17 \%$ reduction in hiatal hernia operations, $11 \%$ reduction in urgent cholecystectomies, $6 \%$ reduction in urgent appendectomies, $17 \%$ reduction in urgent inguinal hernia repairs, and $15 \%$ reduction in colectomies for colon cancer.

\section{Discussion}

This retrospective database study of 559 US hospitals demonstrated a significant reduction in all common GI operations evaluated except for gastrectomies and esophagectomies for cancer. The volume of all operations was lowest in April 2020, with cases increasing from May to June 2020. In June 2020, the GI operations evaluated were reduced by 6\%-17\%, compared to 24\%-98\% in April.

The volume of the elective cases of (delete evaluated, including) bariatric surgery and surgery for hiatal hernias (delete,) decreased the most (96\%-98\% reduction). Both elective operations significantly decreased in top COVID quartile hospitals than (delete and) in hospitals with fewer COVID cases. These cases were likely reduced, especially in the early stages of the pandemic, for many reasons. First, these are considered elective cases and prioritized lower than more time-sensitive operations such as cancer operations. In the vast majority of cases, both operations require postoperative hospital admission, therefore, using more resources than other elective operations. Additionally, these procedures are primarily done laparoscopically, which is considered an aerosolizing procedure [8]. Especially in the early pandemic, COVID-19 testing was significantly limited, restricting availability for routine preoperative testing in asymptomatic patients. Therefore, There was (delete, therefore,) a risk of untested surgical patients with asymptomatic COVID-19 infections exposing surgical staff. Additionally, obese patients undergoing bariatric surgery by definition have an underlying condition that increases their risk for severe COVID-19 infection, making hospital admission during the pandemic higher risk [5, 9].

Interestingly, the urgent and emergent operations evaluated also significantly decreased during the COVID-19 pandemic. Urgent cholecystectomies and inguinal hernia repairs saw a moderate reduction ( $40 \%-42 \%$ reduction), and urgent appendectomies were the least impacted, with a $24 \%$ reduction. Because these are not scheduled operations, the reduction of urgent and emergent cases during the pandemic cannot be attributed to the cancelation of elective operations. 
3000

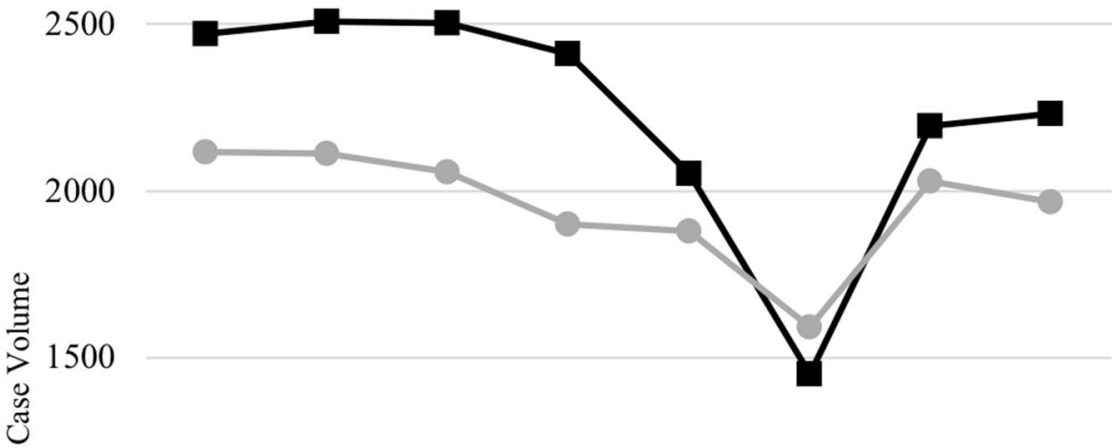

1000 $\rightarrow$ Cholecystectomy

- Appendectomy

- Inguinal Hernia

500

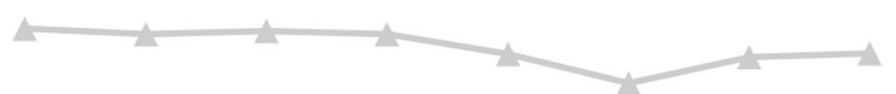

0

$\begin{array}{cccccccc}\text { Nov } & \text { Dec } & \text { Jan } & \text { Feb } & \text { Mar } & \text { Apr } & \text { May } & \text { Jun } \\ 2019 & 2019 & 2020 & 2020 & 2020 & 2020 & 2020 & 2020\end{array}$

Month

Fig. 2 Operative volume by month for urgent/emergent operations

There have been reports from Italy and England showing that there were significant declines in admissions for acute coronary syndromes during the height of the pandemic [10, 11]. This suggests that patients with urgent medical issues delayed seeking medical care during the pandemic, likely (delete possibly) due to fear of contracting COVID-19 at the hospital.

Of the cancer operations evaluated in this study, only colectomies for colon cancer saw a significant reduction during the COVID-19 pandemic (39\% reduction). Gastrectomies and esophagectomies for cancer had their lowest case volumes in April 2020, but differences between months were not statistically significant. Cancer cases have been some of the most difficult to triage. This is especially true for those hospitals hit hard by the pandemic, who were forced to conserve resources more aggressively. In our study, (delete the) cancer operations had the largest differences in volume reduction between the hospitals in the top COVID quartile and hospitals with fewer COVID cases. Colon cancer operations declined twice as much at hospitals in the top COVID quartile compared to hospitals with fewer COVID cases (53\% vs. $27 \%$ reduction, respectively). While the volume of gastrectomies overall did not significantly change by month, there was a significant $32 \%$ reduction in gastrectomies for cancer in hospitals among the top COVID quartile. There are a few potential explanations as to why operative volume for colon cancer significantly declined during the pandemic while operative volume for esophageal and gastric cancers did not. The majority of patients diagnosed with non-metastatic colon cancer proceed to surgery while the majority of patients diagnosed with gastric and esophageal cancers undergo neoadjuvant therapy prior to surgical treatment. Elective colonoscopies were considered the lowest acuity tier by the American College of Surgeons and largely were not performed at the height of the pandemic [5]. It is possible that this lead to a decline in colon cancer diagnoses during the COVID-19 pandemic and, in turn, contributed to the decline in colon cancer operations. In addition, the treatment of esophageal and gastric cancers was likely prioritized more than colon cancers during the pandemic because they tend to be more aggressive. Sud et al. evaluated the survival impact of delaying cancer operations. They found that delaying gastric and esophageal cancers led to a greater reduction in survival than delaying colon cancer surgery [12]. 
1000

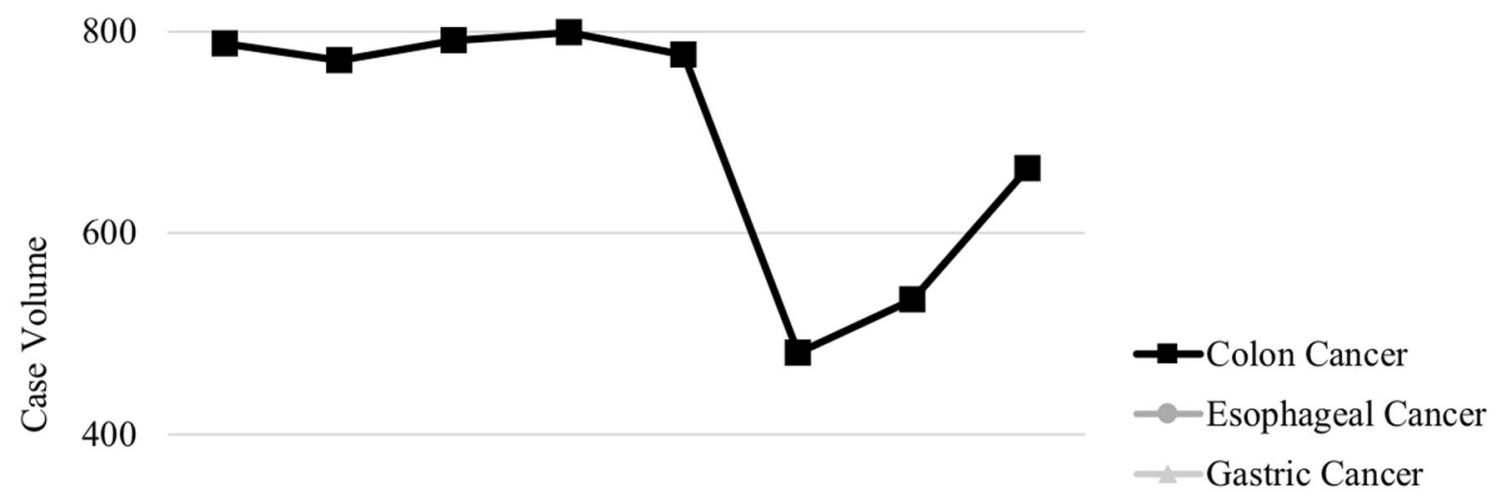

200

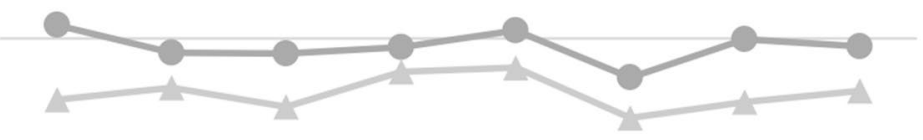

0

$\begin{array}{cccccccc}\text { Nov } & \text { Dec } & \text { Jan } & \text { Feb } & \text { Mar } & \text { Apr } & \text { May } & \text { Jun } \\ 2019 & 2019 & 2020 & 2020 & 2020 & 2020 & 2020 & 2020 \\ \text { Month }\end{array}$

Fig. 3 Operative volume by month for cancer operations

A publication in the Annals of Internal Medicine offered a tool to help prioritize cancer treatment during the pandemic where researchers separated certain cancers based on their risk of progression with treatment delay. Colon cancer without imminent obstruction was considered intermediate risk, with an acceptable delay of 3 months [13]. Delaying surgery for colon cancer does, however, still come with risks. Larson et al. described a possible excess of over 30,000 American deaths over the next 5 years if surgery for colon cancer is delayed more than 4 months.

This study has several limitations. As a retrospective database study, there is an inherent selection bias and risk of ICD coding errors. ICD-10 procedure codes are also specific to inpatients, so data about patients undergoing outpatient procedures are not captured in this study. This is why we chose to examine the specific GI operations in this study, as they are all done primarily in the inpatient setting. Another limitation was that our study likely under-reported the number of coronavirus cases at each hospital because the ICD-10 code for COVID-19 was not in use until March (delete April). In addition, our study focused on the first surge of the pandemic, which does not tell the whole story of the implications of the COVID-19 pandemic on operative volume. While we show that operative volume increased in May and June, it cannot be assumed that this trend of recovery has continued since then because of the additional surges seen in July 2020 and January 2021.

The COVID-19 pandemic has changed how healthcare is delivered in the US. These changes were experienced by not only those actually infected with the novel coronavirus, but also surgical patients who had their operations canceled or delayed. We show a significant reduction in volume of common GI procedures performed during the first surge of the pandemic, including elective operations, urgent/emergent operations, and some cancer operations. While our study highlights the decrease in operative volume during the initial surge, the full implications of the delay in surgical care during the COVID-19 pandemic are yet to be seen. Moving forward, it is important to continue to quantify and characterize the potentially detrimental consequences of the delayed surgical care during the pandemic, and the repercussions of a potential backlog.

Acknowledgements None. 
Funding No funds were granted to perform this study.

Data availability The information contained in this article was based on the clinical database provided by Vizient.

\section{Declarations}

Disclosures Dr. Brian R. Smith is an educational consultant for Stryker Endoscopy. Dr. Ninh Nguyen reported serving as a speaker for Olympus and Endogastric Solutions. Drs. Purdy and Hohmann have no conflicts of interest or financial ties to disclose.

\section{References}

1. CDC COVID Data Tracker. (2020) In: Centers Dis. Control Prev. https://covid.cdc.gov/covid-data-tracker/. Accessed 21 Jan 2021

2. COVID-19 Emergency Declaration. (2020) In: Fed. Emerg. Manag. Agency. https://www.fema.gov/news-release/20200726/ covid-19-emergency-declaration. Accessed 5 Oct 2020

3. COVID-19: Recommendations for Management of Elective Surgical Procedures. (2020) In: Am. Coll. Surg. https://www.facs.org/ covid-19/clinical-guidance/elective-surgery. Accessed 5 Oct 2020

4. Nepogodiev D, Bhangu A, Glasbey JC et al (2020) Mortality and pulmonary complications in patients undergoing surgery with perioperative SARS-CoV-2 infection: an international cohort study. Lancet 396:27-38. https://doi.org/10.1016/S0140-6736(20) 31182-X

5. COVID-19: Guidance for Triage of Non-Emergent Surgical Procedures. In: Am. Coll. Surg. https://www.facs.org/covid-19/clini cal-guidance/triage. Accessed 9 Oct 2020

6. Chang EI, Liu JJ (2020) Flattening the curve in oncologic surgery: Impact of Covid-19 on surgery at tertiary care cancer center. J Surg Oncol 122:602-607. https://doi.org/10.1002/jso.26056

7. Vizient Inc. Co website. https://www.vizientinc.com/. Accessed 1 Aug 2020

8. Francis N, Dort J, Cho E, Feldman L, Keller D, Lim R, Mikami D, Phillips E, Spaniolas K, Tsuda S, Wasco K, Arulampalam T, Sheraz M, Morales S, Pietrabissa A, Asbun H, Pryor A (2020)
SAGES and EAES recommendations for minimally invasive surgery during COVID-19 pandemic. Surg Endosc 34:2327-2331. https://doi.org/10.1007/s00464-020-07565-w

9. Lighter J, Phillips M, Hochman S, Sterling S, Johnson D, Francois F, Stachel A (2020) Obesity in Patients Younger Than 60 Years Is a Risk Factor for COVID-19 Hospital Admission. Clin Infect Dis 71:896-897. https://doi.org/10.1093/cid/ciaa415

10. De Filippo O, D’Ascenzo F, Angelini F, Bocchino PP, Conrotto F, Saglietto A, Secco GG, Campo G, Gallone G, Verardi R, Gaido L, Iannaccone M, Galvani M, Ugo F, Barbero U, Infantino V, Olivotti L, Mennuni M, Gili S, Infusino F, Vercellino M, Zucchetti O, Casella G, Giammaria M, Boccuzzi G, Tolomeo P, Doronzo B, Senatore G, Grosso Marra W, Rognoni A, Trabattoni D, Franchin L, Borin A, Bruno F, Galluzzo A, Gambino A, Nicolino A, Truffa Giachet A, Sardella G, Fedele F, Monticone S, Montefusco A, Omedè P, Pennone M, Patti G, Mancone M, De Ferrari GM (2020) Reduced Rate of Hospital Admissions for ACS during Covid-19 Outbreak in Northern Italy. N Engl J Med 383:88-89. https://doi.org/10.1056/NEJMc2009166

11. Mafham MM, Spata E, Goldacre R, Gair D, Curnow P, Bray M, Hollings S, Roebuck C, Gale CP, Mamas MA, Deanfield JE, de Belder MA, Luescher TF, Denwood T, Landray MJ, Emberson JR, Collins R, Morris EJA, Casadei B, Baigent C (2020) COVID19 pandemic and admission rates for and management of acute coronary syndromes in England. Lancet 396:381-389. https://doi. org/10.1016/S0140-6736(20)31356-8

12. Sud A, Jones ME, Broggio J, Loveday C, Torr B, Garrett A, Nicol DL, Jhanji S, Boyce SA, Gronthoud F, Ward P, Handy JM, Yousaf N, Larkin J, Suh Y-E, Scott S, Pharoah PDP, Swanton C, Abbosh C, Williams M, Lyratzopoulos G, Houlston R, Turnbull C (2020) Collateral damage: the impact on outcomes from cancer surgery of the COVID-19 pandemic. Ann Oncol 31:1065-1074. https:// doi.org/10.1016/j.annonc.2020.05.009

13. Kutikov A, Weinberg DS, Edelman MJ, Horwitz EM, Uzzo RG, Fisher RI (2020) A War on Two Fronts: Cancer Care in the Time of COVID-19. Ann Intern Med 172:756-758. https://doi.org/10. 7326/M20-1133

Publisher's Note Springer Nature remains neutral with regard to jurisdictional claims in published maps and institutional affiliations. 